\title{
Composition of mixed adsorption layers and micelles in solutions of sodium dodecyl sulfate and dodecyl acid diethanol amide
}

\author{
J.K. Angarska ${ }^{a}$,, K.D. Tachev ${ }^{a}$, N.D. Denkov ${ }^{b}$ \\ a Faculty of Natural Sciences, University of Shoumen, Shoumen 9712, Bulgaria \\ ${ }^{\mathrm{b}}$ Faculty of Chemistry, Laboratory of Chemical Physics and Engineering, University of Sofia, 1164 Sofia, Bulgaria
}

Received 17 June 2003; accepted 18 November 2003

\begin{abstract}
The surface tension isotherms of dodecyl acid diethanole amide (DADA), sodium dodecyl sulfate (SDS) and their mixtures at different molar ratios are measured by the Wilhelmy-plate method. The isotherms are analyzed by the theory of nonideal interactions in binary surfactant mixtures (NIBM) and by the nonideal multicomponent mixed micelle model (NMMM). The molecular interaction parameters (MIP) on the interface, $\beta_{\mathrm{S}}$, and in the micelles, $\beta_{\mathrm{M}}$, are determined by the NIBM method. By using the Ingram's modification of this method, we calculate the compositions of the mixed adsorption layers and of the micelles, as functions of DADA and SDS concentrations both below and above the critical micelle concentration (CMC). It is shown that the DADA molecules prevail on the surface and in the micelles $(>50 \%)$ at concentrations around $\mathrm{CMC}$ even when DADA presents a small fraction in the surfactant mixture $(5 \mathrm{~mol} \%)$. This result is explained by the much higher surface activity of DADA in comparison with SDS. At concentrations, which are about 1 order of magnitude above the CMC, the composition of the micelles approaches the composition of the total surfactant mixture. The composition of the surfactant adsorption layer also becomes much closer to the composition of the total surfactant mixture at concentrations well above the CMC. The results for the micelles composition at moderate and high molar fractions of DADA in the surfactant mixture (above 10 mol\%), obtained by NMMM and by NIBM methods, agree well with each other. A discrepancy between the results obtained by these two models is found at low DADA fraction in the mixture, and this discrepancy is explained by the presence of traces of dodecanol (DOH) in the used SDS sample.
\end{abstract}

(c) 2003 Elsevier B.V. All rights reserved.

Keywords: Mixed surfactant solutions; Molecular interaction parameters; Surface tension isotherms; Mixed adsorption layers; Micelle composition

\section{Introduction}

Surfactant mixtures are widely used in practice as foam and emulsion stabilizers due to their superior properties as compared to pure surfactants. The synergistic action of surfactant mixtures is used in detergency, hair-care, body-care, house-hold, and many other surfactant-based products [1-3]. For example, it was found experimentally that the critical micellization concentration (CMC) for surfactant mixtures is often lower than that of the individual components, which decreases the irritation effect of the surfactant solutions on skin and eyes. Usually, this synergism is explained by the presence of strong specific interactions between the surfactant molecules in the mixed adsorption layers and in the mixed micelles [4].

\footnotetext{
* Corresponding author. Tel.: +359-54-830-495x272; fax: +359-54-830-371.

E-mail address: angarska@shu-bg.net (J.K. Angarska).
}

A co-surfactant, that is used as an additive and improves significantly the foaming properties of the major surfactant, is often called a "foam booster." Data showing that dodecyl acid diethanole amide (DADA) plays the role of a foam booster for the anionic surfactant sodium dodecylpolyoxyethylene-3 sulfate (SDP3S) were presented in [5] (note that the abbreviation LADA, instead of DADA, was used for the same substance in [5]). The detailed mechanism, which leads to the foam boosting effect of DADA is not entirely clear, but it might be expected that this mechanism is related to a specific interaction between the DADA and SDP3S molecules in the mixed adsorption layers (a quantitative characterization of this interaction was not attempted in [5]).

In the present article we analyze the interactions between sodium dodecyl sulfate (SDS) and DADA molecules in the mixed adsorption layers and in the mixed micelles. SDS was chosen for this study, because this anionic surfactant is widely used in practical applications as foaming agent and 
emulsifier. SDS has relatively high CMC and, for this reason, it is usually applied in combination with other surface active additives (boosters), such as DADA or various betaines [2].

The DADA molecules are uncharged at neutral $\mathrm{pH}$, but they can participate in a donor-acceptor bond through the free electron pair of the $\mathrm{N}$-atom and bind protons at low $\mathrm{pH}$ (rendering a positive charge). According to [2], a positive synergistic action could be expected for co-surfactants of such chemical structure and anionic surfactants, like SDS. The pronounced foam boosting effect of DADA with respect to SDP3S solutions [5] suggests that DADA probably has a negative "molecular interaction parameters" (MIP) with SDP3S, and one may expect that the same is true for SDS, as well.

According to $[1-4,6,7]$ the molecular interaction parameters in binary surfactant mixtures could be determined from equilibrium surface tension isotherms by using the theory of nonideal interactions in binary surfactant mixtures (NIBM). Based on this theory, approaches for determination of the composition of mixed adsorption layers and mixed micelles were proposed in $[1,8]$. Alternatively, the nonideality of the solution properties, caused by the interactions between various surfactant components in mixed micelles, could be analyzed by the regular solutions theory-this approach is implemented in the so-called nonideal multicomponent mixed micelle model (NMMM) developed in [9]. The composition of the mixed micelles and the concentrations of the individual monomers in the surfactant solution can be calculated by this model for multicomponent mixtures, containing even more than two components.

In the current study we present data for the equilibrium surface tension isotherms of SDS, DADA, and their mixtures, at different molar fractions of DADA in the solutions. The major aims of the study are:

(i) to analyze the experimental data by the NIBM and NMMM and to determine the composition of the adsorption layers and of the micelles, along with the respective molecular interaction parameters (on the interface and in the micelles);

(ii) to compare the results for the micelle composition determined by the two methods and to evaluate how the dodecanol $(\mathrm{DOH})$, which is present in the used SDS sample of technical grade, affects some of the properties of the mixed SDS/DADA solutions.

\section{Materials and methods}

The used SDS and DADA were both commercial grade products from KAO Co., Tokyo, Japan. In one series of experiments, dodecanol, product of Sigma Chemical Co., St. Louis, MO, USA, was used as an additive.

Several types of solutions were studied: solutions of the individual surfactants SDS, DADA, and DOH; solutions of the mixtures SDS/DADA containing 0.05, 0.09, 0.2 and 0.7 molar parts of DADA; and the mixture DADA/DOH containing 0.0015 molar part of DOH. All solutions containing SDS were used immediately after their preparation to minimize the possible effect of SDS hydrolysis on the solution properties [10].

The Wilhelmy-plate method was used for measuring the surface tension of the solutions, $\sigma$. The equilibrium values of $\sigma$ were measured, by using a frosted glass plate, $6 \mathrm{~h}$ after the solution was poured in a PTFE container. To prevent the possible strong adsorption of DOH on the PTFE walls of the container (which could result in an uncontrolled reduction of DOH concentration in the solution), a glass vessel was used when DOH and DADA/DOH solutions were studied.

All measurements were carried out at $25 \pm 0.5^{\circ} \mathrm{C}$.

\section{Theoretical background-approaches for evaluation of the composition of mixed adsorption layers and micelles}

\subsection{Model of nonideal interactions in binary surfactant mixtures (NIBM)}

\subsubsection{NIBM model of Rosen}

The measured surface tension isotherms (see Fig. 1) were analyzed by the NIBM model developed by Rosen et al. $[1,6,7]$. According to this model, the composition of the adsorption layers for a two-component mixture can be determined by Eq. (1), whereas the micelles composition at the $\mathrm{CMC}$ can be determined from Eq. (2):

$$
\begin{aligned}
& \frac{X_{1 \mathrm{~S}}^{2} \ln \left(\alpha C_{12} / X_{1 \mathrm{~S}} C_{1}\right)}{\left(1-X_{1 \mathrm{~S}}\right)^{2} \ln \left[(1-\alpha) C_{12} /\left(1-X_{1 \mathrm{~S}}\right) C_{2}\right]}=1 \\
& \frac{X_{1 \mathrm{M}}^{2} \ln \left(\alpha C_{12 \mathrm{M}} / X_{1 \mathrm{M}} C_{1 \mathrm{M}}\right)}{\left(1-X_{1 \mathrm{M}}\right)^{2} \ln \left[(1-\alpha) C_{12 \mathrm{M}} /\left(1-X_{1 \mathrm{M}}\right) C_{2 \mathrm{M}}\right]}=1
\end{aligned}
$$

where $\alpha$ is the molar fraction of surfactant 1 (DADA) in the bulk solution; the molar part of surfactant 2 (i.e. of SDS) is

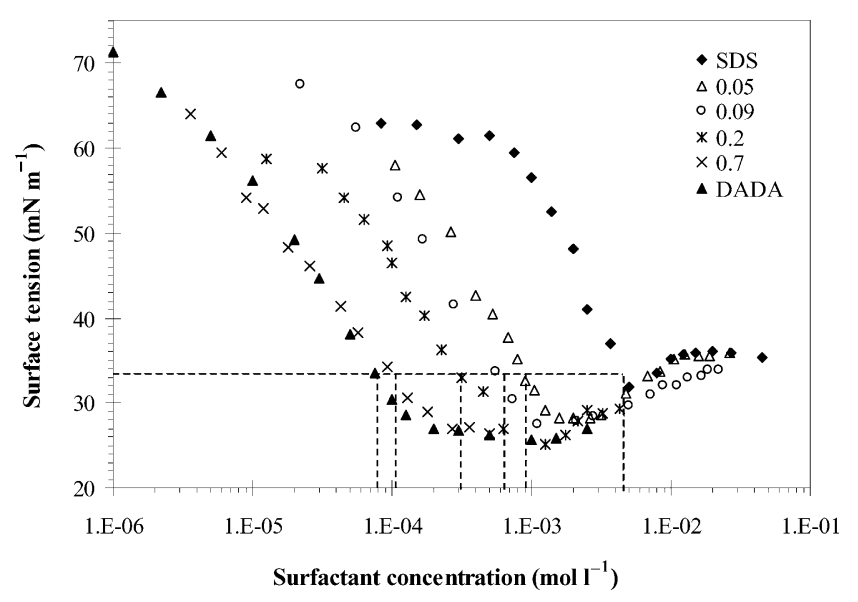

Fig. 1. Equilibrium surface tension, $\sigma$, of SDS, DADA and their mixed solutions (containing different molar fractions of DADA: 0.05, 0.09, 0.2 and 0.7$)$ vs. total surfactant concentration, $C_{\mathrm{t}}$. 
Table 1

Experimental and calculated values for mixed DADA/SDS adsorption layers and micelles

\begin{tabular}{|c|c|c|c|c|c|c|c|}
\hline \multirow[t]{2}{*}{$\alpha$} & \multicolumn{3}{|c|}{ Experimental data } & \multicolumn{4}{|c|}{ Calculated by the method of Rosen, Eqs. (1)-(4) } \\
\hline & $C_{\min }(\mathrm{mM})$ & $\mathrm{CMC}(\mathrm{mM})$ & $\begin{array}{l}C_{1},(\mathrm{mM}) \text { at } \\
\sigma=33.5 \mathrm{mN} \mathrm{m}^{-1}\end{array}$ & $X_{1 \mathrm{~S}}$ & $X_{1 \mathrm{M}}$ & $\beta_{\mathrm{S}}$ & $\beta_{\mathrm{M}}$ \\
\hline 0.00 & 5.00 & 12.30 & 4.52 & - & - & - & - \\
\hline 0.05 & 2.00 & 12.00 & 0.870 & 0.670 & 0.651 & -1.31 & 0.20 \\
\hline 0.09 & 1.50 & 8.80 & 0.611 & 0.774 & 0.887 & -1.02 & 1.12 \\
\hline 0.20 & 1.25 & 2.50 & 0.304 & 0.843 & 0.876 & -1.51 & $\mathrm{a}$ \\
\hline 0.70 & - & 0.27 & 0.106 & 0.991 & 0.990 & -0.29 & $\mathrm{a}$ \\
\hline 1.00 & - & 0.15 & 0.075 & - & - & - & - \\
\hline
\end{tabular}

$\alpha$ is the molar fraction of DADA in the surfactant mixture; $C_{\min }$, concentration corresponding to the minimum in the surface tension isotherm (see Fig. 1); CMC, critical micelle concentration; $C_{1}$, concentration of DADA in the surfactant mixture at $\sigma=33.5 \mathrm{mN} \mathrm{m}^{-1} ; X_{1 \mathrm{~S}}$, molar fraction of DADA in the adsorption layer at $\sigma=33.5 \mathrm{mN} \mathrm{m}^{-1} ; X_{1 \mathrm{M}}$, molar fraction of DADA in the mixed micelles at CMC; $\beta_{\mathrm{S}}$ and $\beta_{\mathrm{M}}$, DADA/SDS interaction parameters for the adsorption layer and the micelles, respectively.

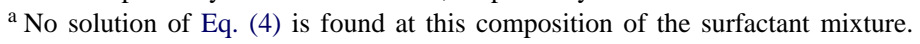

equal to $(1-\alpha) ; X_{1 S}$, molar part of surfactant 1 in the mixed adsorption layer; $X_{1 \mathrm{M}}$, molar part of component 1 in the mixed micelles; $C_{1}, C_{2}$, and $C_{12}$, molar concentrations of the solutions containing pure surfactants 1,2 and their mixture $(1+2)$, respectively, corresponding to a given surface tension value (close to $\mathrm{CMC}$, in the linear part of the surface tension isotherm). In our experiments, we determined $C_{1}, C_{2}$, and $C_{12}$, which correspond to surface tension $\sigma=33.5 \mathrm{mN} \mathrm{m}^{-1}$ (see Fig. 1). The quantities $C_{1 \mathrm{M}}, C_{2 \mathrm{M}}$, and $C_{12 \mathrm{M}}$ are the CMCs of the individual surfactants 1,2 and of their mixture (at a certain value of $\alpha$ ).

From Eqs. (1) and (2), the values of $X_{1 \mathrm{~S}}$ and $X_{1 \mathrm{M}}$ were determined by iterative numerical procedure, by using the values of $C_{i}$ and $C_{i \mathrm{M}}(i=1,2)$, which were determined experimentally from the surface tension isotherms, see Table 1.

The interactions between the two components in the surfactant mixture can be characterized by the so-called molecular interaction parameters, $\beta_{\mathrm{S}}$ and $\beta_{\mathrm{M}}$, for the adsorption layer and for the micelles, respectively. If one assumes that $\beta_{\mathrm{S}}$ and $\beta_{\mathrm{M}}$ do not depend on the total surfactant concentration and on the relative fraction of the two components in the mixture, these parameters can be evaluated by the equations $[7,11]$ :

$\beta_{\mathrm{S}}=\frac{\ln \left(\alpha C_{12} / X_{1 \mathrm{~S}} C_{1}\right)}{\left(1-X_{1 \mathrm{~S}}\right)^{2}}$

$\beta_{\mathrm{M}}=\frac{\ln \left(\alpha C_{12 \mathrm{M}} / X_{1 \mathrm{M}} C_{1 \mathrm{M}}\right)}{\left(1-X_{1 \mathrm{M}}\right)^{2}}$

Negative values of the interaction parameters indicate net attractive interaction between the surfactant molecules. If the magnitude of $\beta_{\mathrm{S}}$ is sufficiently large, the surface tension of the surfactant mixture could be lower than the surface tensions of the individual surfactants at the same total surfactant concentration (so-called "positive synergism"). With respect to the formation of mixed micelles, the positive synergism exhibits as lowering the $\mathrm{CMC}$ of the mixture below the CMCs of the individual surfactants. As shown by Rosen [1], the conditions for a synergism in the surface tension and in the formation of mixed micelles can be expressed by
Eqs. $\left(3^{\prime}\right)$ and $\left(4^{\prime}\right)$, respectively,

$$
\begin{aligned}
& \left|\beta_{\mathrm{S}}\right|>\left|\ln \left(\frac{C_{1}}{C_{2}}\right)\right| \\
& \left|\beta_{\mathrm{M}}\right|>\left|\ln \left(\frac{C_{1 \mathrm{M}}}{C_{2 \mathrm{M}}}\right)\right|
\end{aligned}
$$

\subsubsection{Modification of the NIBM method by Ingram [8]}

The measured surface tension isotherms were analyzed also by the modification of the NIBM method, suggested by Ingram [8], which is based on the following set of two equations for the composition of the mixed adsorption layer:

$X_{1 \mathrm{~S}} \exp \left[\beta_{\mathrm{S}}\left(1-X_{1 \mathrm{~S}}\right)^{2}\right]=\frac{C_{1}}{\Phi_{1,0}(\sigma)}$

$\left(1-X_{1 \mathrm{~S}}\right) \exp \left[\beta_{\mathrm{S}} X_{1 \mathrm{~S}}^{2}\right]=\frac{C_{2}}{\Phi_{2,0}(\sigma)}$

where $\beta_{\mathrm{S}}$ is the interaction constant for the adsorption layer (equivalent to the interaction parameter in the model of Rosen); $X_{1 \mathrm{~S}}$, molar part of surfactant 1 (DADA) in the mixed adsorption layer; $C_{1}$ and $C_{2}$, monomer concentrations in the bulk solution (close to, but below CMC of the mixture); $C_{1}=\alpha C_{\mathrm{t}}$ and $C_{2}=(1-\alpha) C_{\mathrm{t}}$, where $C_{\mathrm{t}}$ is the total molar concentration of the two surfactants; $\Phi_{1,0}(\sigma)$ and $\Phi_{2,0}(\sigma)$, so-called "tension functions" for the solutions of the individual surfactants. Close to CMC, these functions are assumed [8] to obey the equation $\Phi=\exp \left(K^{\prime}-K^{\prime \prime} \sigma\right)$, where $K^{\prime}$ and $K^{\prime \prime}$ are constants characterizing the used surfactant. The constants $K_{1}^{\prime}, K_{1}^{\prime \prime}$ and $K_{2}^{\prime}, K_{2}^{\prime \prime}$ for DADA and SDS, respectively, were determined from the surface tension isotherms of the individual surfactants (see Fig. 2). The following values were obtained: $K_{1}^{\prime}=-5.7328, K_{1}^{\prime \prime}=0.1081, K_{2}^{\prime \prime}=$ -2.9442 , and $K_{2}^{\prime \prime}=0.0717$.

The micelles composition at CMC was evaluated by the following set of equations [8]:

$$
\begin{aligned}
& X_{1 \mathrm{M}} \exp \left[\beta_{\mathrm{M}}\left(1-X_{1 \mathrm{M}}\right)^{2}\right]=\frac{C_{1}}{C_{1 \mathrm{M}}} \\
& \left(1-X_{1 \mathrm{M}}\right) \exp \left[\beta_{\mathrm{M}} X_{1 \mathrm{M}}^{2}\right]=\frac{C_{2}}{C_{2 \mathrm{M}}}
\end{aligned}
$$




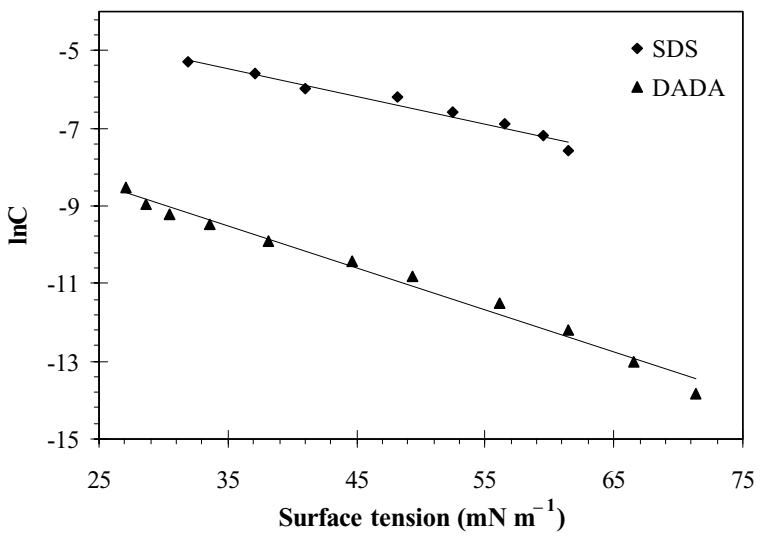

Fig. 2. Plots of $\ln C$ vs. surface tension, used for determination of the adsorption constants $K_{1}^{\prime}, K_{1}^{\prime \prime}$ and $K_{2}^{\prime}, K_{2}^{\prime \prime}$ of DADA and SDS, respectively (see Eq. (5) and the comments below it).

where $\beta_{\mathrm{M}}$ is the interaction constant in the micelle; $X_{1 \mathrm{M}}$, molar part of surfactant 1 in the mixed micelle; $C_{1 \mathrm{M}}$ and $C_{2 \mathrm{M}}$, CMCs of the individual surfactants.

In fact, Eqs. (5) and (6) in the Ingram's model are equivalent to Eqs. (1) and (3) in the Rosen's model. That is why, the calculated values for the interaction parameters, $\beta_{\mathrm{S}}$ and $\beta_{\mathrm{M}}$, and the compositions of the surface layer at $\sigma=$ $33.5 \mathrm{mN} \mathrm{m}^{-1}$ and of the micelles at $\mathrm{CMC}$, determined by the two models, were exactly the same (see Table 1). However, the approach of Ingram [8] allows one to calculate the surface tension isotherms and the composition of the adsorption layers for surfactant mixtures at arbitrary surfactant concentrations below and above the CMC. The composition of the mixed micelles can be also calculated by this method.

The procedure of calculations is the following: The value of $\beta_{\mathrm{S}}$ (determined from the data below CMC) is substituted into Eq. (5) to calculate $\sigma$ and $X_{1 \mathrm{~S}}$ at an arbitrary value of $C_{\mathrm{t}}$ below CMC. Above CMC, the composition of the micelles, $X_{1 \mathrm{M}}$, is determined by solving the following equation [8]:

$P X_{1 \mathrm{M}}^{2}+\left(C_{\mathrm{t}}-P\right) X_{1 \mathrm{M}}-\alpha C_{\mathrm{t}}=0$

where, by definition $P=C_{2 \mathrm{M}} \exp \left[\beta_{\mathrm{M}} X_{1 \mathrm{M}}^{2}\right]-C_{1 \mathrm{M}} \exp \left[\beta_{\mathrm{M}}\right.$ $\left(1-X_{1 \mathrm{M}}\right)^{2}$ ]. The values of $X_{1 \mathrm{M}}$, calculated from Eq. (7) are substituted into Eq. (6) to yield the concentration of the monomers, $C_{1}$ and $C_{2}$, which in turn are inserted into Eq. (5) to determine the surface tension, $\sigma$, and the composition of the adsorption layer, $X_{1 \mathrm{~S}}$, above the CMC.

\subsection{Nonideal multicomponent mixed micelle model (NMMM) [9]}

This model was used to evaluate the composition of the mixed micelles in the SDS/DADA solutions, taking into account the presence of DOH in the used SDS sample. The NMMM presents a generalization of the pseudo-phase separation model for treatment of multicomponent nonideal micelle systems. The approach allows one to determine the micelle composition, as well as the concentrations of the individual monomers. According to the NMMM, the mole fraction, $X_{i \mathrm{M}}$, of the $i$-th component in the mixed micelles can be expressed as:

$X_{i \mathrm{M}}=\frac{\alpha_{i} C_{\mathrm{t}}}{C_{i} f_{i}-C_{j} f_{j}+\alpha_{j} C_{\mathrm{t}} / X_{j \mathrm{M}}}$

where $\alpha_{i}$ and $\alpha_{j}$ are the mole fractions of any two components $i$ and $j$ in the solution; $f_{i}$ and $f_{j}$, the respective activity coefficients.

According to the theory of regular solutions, one can use the following expression for the activity coefficients of the various components:

$$
\begin{aligned}
\ln f_{i}= & \sum_{j=1(j \neq i)}^{n} \beta_{i j} X_{j \mathrm{M}}^{2} \\
& +\sum_{\substack{j=1 \\
j \neq i \neq k)}}^{n} \sum_{k=1}^{j-1}\left(\beta_{i j}+\beta_{i k}-\beta_{j k}\right) X_{j \mathrm{M}} X_{k \mathrm{M}}
\end{aligned}
$$

The parameters $\beta_{i j}$ in Eq. (9) are constants, characterizing the pairwise interactions in the mixed micelles. These parameters can be expressed through the interaction energies, $W_{i j}$, between the various molecular species in the micelles and can be determined from experimental surface tension isotherms of the respective binary surfactant mixtures (see Eq. (4)).

If the parameters $\beta_{i j}$ are known, the problem for determination of the composition of the mixed micelles, $X_{i \mathrm{M}}$, and the monomer concentrations in the surfactant solution, $C_{i}$, requires one to solve the set of equations, obtained by substituting Eq. (9) into Eq. (8) for the various components, and by using the natural constraint $\sum_{i=1}^{n} X_{i \mathrm{M}}=1$. For the studied three-component system (DADA, SDS, DOH) one derives the following set of equations:

$$
\ln f_{1}=\beta_{12} X_{2 \mathrm{M}}^{2}+\beta_{13} X_{3 \mathrm{M}}^{2}+\left(\beta_{12}+\beta_{13}-\beta_{23}\right) X_{2 \mathrm{M}} X_{3 \mathrm{M}}
$$

$\ln f_{2}=\beta_{12} X_{1 \mathrm{M}}^{2}+\beta_{23} X_{3 \mathrm{M}}^{2}+\left(\beta_{12}+\beta_{23}-\beta_{13}\right) X_{1 \mathrm{M}} X_{3 \mathrm{M}}$

$\ln f_{3}=\beta_{13} X_{1 \mathrm{M}}^{2}+\beta_{23} X_{2 \mathrm{M}}^{2}+\left(\beta_{13}+\beta_{23}-\beta_{12}\right) X_{1 \mathrm{M}} X_{2 \mathrm{M}}$

$X_{1 \mathrm{M}}=\frac{\alpha_{1} C_{\mathrm{t}}}{C_{1} f_{1}-C_{2} f_{2}+\left(\alpha_{2} C_{\mathrm{t}} / X_{2 \mathrm{M}}\right)}$

$X_{2 \mathrm{M}}=\frac{\alpha_{2} C_{\mathrm{t}}}{C_{2} f_{2}-C_{3} f_{3}+\left(\alpha_{3} C_{\mathrm{t}} / X_{3 \mathrm{M}}\right)}$

where the indices 1,2 , and 3 refer to DADA, SDS, and $\mathrm{DOH}$, respectively.

We solved numerically the set of Eqs. (10) and (11) by iteration steps-the values of $X_{1 \mathrm{M}}$ and $X_{2 \mathrm{M}}$ were calculated 
by using Eq. (11) $\left(X_{3 \mathrm{M}}=1-X_{1 \mathrm{M}}-X_{2 \mathrm{M}}\right)$ until the quadratic difference between the values calculated in two consecutive steps converged to a pre-described precision.

\section{Experimental results and discussion}

\subsection{Surface tension isotherms}

The measured surface tension isotherms of the individual surfactants SDS and DADA, and of their mixtures at DADA molar fractions $\alpha=0.05,0.09,0.2$, and 0.7, are plotted in Fig. 1. As seen from this figure, a minimum in the isotherm of SDS is observed at $C_{\min } \approx 5 \times 10^{-3} \mathrm{M}$ $\left(\sigma_{\min }=31.9 \mathrm{mN} \mathrm{m}^{-1}\right)$. After this minimum, the value of $\sigma$ increases and reaches almost a constant value, $\approx 35 \mathrm{mN} \mathrm{m}^{-1}$, at $C_{\mathrm{SDS}} \geq 1.2 \times 10^{-2} \mathrm{M}$. The minimum in the isotherm indicates that the used SDS sample contains traces of DOH as an impurity (see e.g. [10]). We do not expect that DOH was created as a result of SDS hydrolysis in the studied solutions, because we used only fresh SDS solutions (according to [12], the hydrolysis of SDS is rather slow at neutral $\mathrm{pH}$ ). As suggested in [10], the CMC of the surfactant solution was associated with the beginning of the plateau region after the minimum. Thus, $\mathrm{CMC}=1.23 \times 10^{-2} \mathrm{M}$ was accepted for the SDS solutions (without DADA). The values of CMC and of $C_{\min } \approx 5 \times 10^{-3} \mathrm{M}$ for the used SDS solutions are in a reasonably good agreement with the respective values determined in [10] $\left(1.0 \times 10^{-2}\right.$ and $7.5 \times 10^{-3} \mathrm{M}$, respectively) for SDS (98\% purity from Aldrich) solutions containing traces of $\mathrm{DOH}$. The presence of $\mathrm{DOH}$, as an impurity in the used SDS sample, is supported also by the value for the effective surfactant adsorption, $\Gamma_{\mathrm{TOT}}=5.5 \times 10^{-6} \mathrm{~mol} \mathrm{~m}^{-2}$, which was calculated from the slope of the isotherm just below the CMC. The latter value is considerably higher than the value determined by Tajima et al. [13] for pure SDS $\left(\Gamma_{\mathrm{SDS}}=3.2 \times 10^{-6} \mathrm{~mol} \mathrm{~m}^{-2}\right)$.

No minimum was observed in the surface tension isotherm of pure DADA. The obtained value of CMC, $1.5 \times 10^{-4} \mathrm{M}$, is about 2 orders of magnitude lower than the CMC of the SDS solutions, which indicates the significantly higher surface activity of DADA.

One sees from Fig. 1 that the isotherms of the mixed SDS/DADA solutions are placed between the isotherms of the individual surfactants. The shape of the isotherms of the solutions containing 0.05, 0.09 and 0.2 molar parts of DADA resemble that of the SDS solutions (a minimum in the isotherm is observed). The isotherm of the solutions containing 0.7 molar parts of DADA is almost identical to that of pure DADA, which indicates that the adsorption layer in this system was composed exclusively of DADA molecules. The above conclusions are confirmed by the numerical analysis of the isotherms, as well (see Section 4.3 below).

The isotherm of mixed DADA/DOH solutions, containing 0.0015 molar parts of $\mathrm{DOH}$, was also measured to determine the interaction parameter between these two sur-

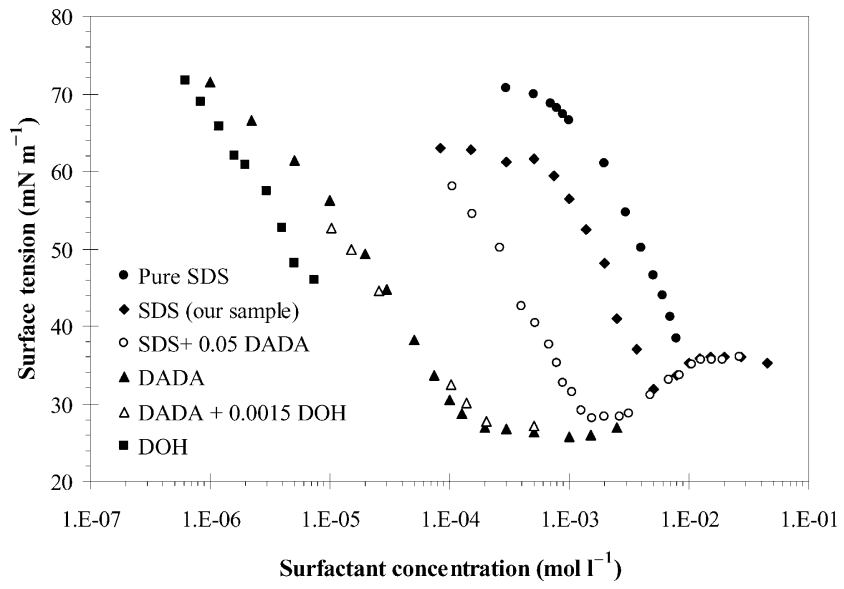

Fig. 3. Equilibrium surface tension isotherms used to estimate the molecular interaction parameters in the micelles $\left(\beta_{i j}\right)$ between: SDS and DOH $\left(\beta_{23}=-1.41\right)$; DADA and SDS $\left(\beta_{12}=+0.20\right)$; DADA and DOH $\left(\beta_{13}=+0.10\right)$. The isotherm of pure SDS is taken from [13], the isotherm of pure $\mathrm{DOH}$ is from [10].

factants, $\beta_{13}$, and this isotherm was found to be very similar to the isotherm of pure DADA (see Fig. 3).

\subsection{Evaluation of the content of dodecanol in the used SDS sample}

For the data analysis by the NMMM method we need to know the amount of all three-components (DADA, SDS, and $\mathrm{DOH}$ ) in the surfactant mixtures. The relative amount of DOH in the used SDS sample was estimated by using the simplifying assumption that the adsorptions of DOH and SDS can be described by Langmuir adsorption isotherm from a binary mixture, and that the area per molecule in the adsorption layer is the same for the two components. Under these assumptions, one derives the following relation:

$\frac{\Gamma_{\mathrm{DOH}}}{\Gamma_{\mathrm{SDS}}}=\frac{k_{\mathrm{DOH}}}{k_{\mathrm{SDS}}} \frac{C_{\mathrm{DOH}}}{C_{\mathrm{SDS}}}$

where $\Gamma_{\mathrm{DOH}}$ and $\Gamma_{\mathrm{SDS}}$ are the adsorptions, while $k_{\mathrm{DOH}}$ and $k_{\mathrm{SDS}}$ are the adsorption constants of DOH and SDS, respectively. Substituting $k_{\mathrm{SDS}}=5 \times 10^{2} \mathrm{M}^{-1}$ and $k_{\mathrm{DOH}}=$ $2.5 \times 10^{5} \mathrm{M}^{-1}$ (taken from [14]), and assuming that $\Gamma_{\mathrm{DOH}}$ can be presented as $\Gamma_{\mathrm{DOH}}=\Gamma_{\mathrm{TOT}}-\Gamma_{\mathrm{SDS}}$ we derive:

$\frac{\Gamma_{\mathrm{TOT}}-\Gamma_{\mathrm{SDS}}}{\Gamma_{\mathrm{SDS}}}=500 \frac{C_{\mathrm{DOH}}}{C_{\mathrm{SDS}}}$

By using $\Gamma_{\text {TOT }}=5.5 \times 10^{-6} \mathrm{~mol} \mathrm{~m}^{-2}$ (see Fig. 4) and $\Gamma_{\mathrm{SDS}}=3.2 \times 10^{-6} \mathrm{~mol} \mathrm{~m}^{-2}$ for pure SDS at $C_{\mathrm{SDS}}=3.2 \times$ $10^{-3} \mathrm{M}$ [13], we estimated $C_{\mathrm{DOH}} \approx 4.9 \times 10^{-6} \mathrm{M}$, i.e. the content of DOH in the used SDS sample is about 0.0015 molar parts. A more refined analysis of the measured surface tension isotherm of our technical SDS by the method of Kralchevsky et al. [15] gave similar result for the molar fraction of DOH in the used SDS sample (0.0012 molar parts). Although this molar fraction of DOH is rather low, we analyzed the $\mathrm{DOH}$ contribution to the composition of the 


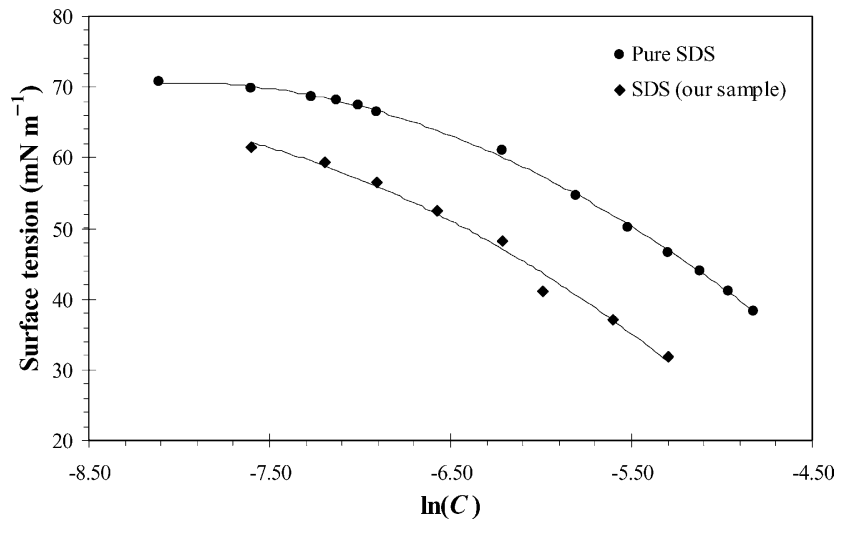

Fig. 4. Surface tension isotherms of pure SDS [13] and of our technical SDS, which were used to estimate the DOH molar fraction in our SDS sample (see Section 4.2).

mixed micelles by the NMMM model, in order to compare the results with those from the NIBM model (Section 4.4).

\subsection{Composition of the mixed adsorption layers and of the mixed micelles, as calculated by NIBM}

The relatively small content of DOH in the SDS samples allows us to ignore its presence in the micelles and in the adsorption monolayers (as a first approximation), and to consider the system as a binary DADA/SDS mixture that can be analyzed by the NIBM model.

The CMC values of the individual SDS and DADA, and of their mixed solutions, as determined from the isotherms (Fig. 1), are presented in Table 1. Based on these data, we solved Eqs. (2) and (4) and determined the interaction parameter, $\beta_{\mathrm{M}}$, and the micelle composition at CMC, $X_{1 \mathrm{M}}$, according to the model of Rosen [1]. Let us note that by using Eq. (6) and the same experimental data, we determined the micelle composition according to the model of Ingram [8] and obtained the same results, because the equations of Rosen [1] and Ingram [8] are equivalent at CMC.

From the surface tension isotherms, we determined the surfactant concentration corresponding to $\sigma=33.5 \mathrm{mN} \mathrm{m}^{-1}$ (see Table 1). These concentrations were used to solve Eqs. (1), (3) and (5), and to obtain the values of $X_{1 \mathrm{~S}}$ and $\beta_{\mathrm{S}}$ in accordance with the models of Rosen [1] and Ingram [8] (see Table 1). We found in these calculations that the value of $\beta_{\mathrm{S}}$ was equal to $-1.28 \pm 0.25$ (for molar fraction of DADA in the mixture $\alpha=0.05,0.09$ and 0.2). At the highest molar fraction of DADA $(\alpha=0.7)$ much lower in magnitude value of $\beta_{\mathrm{S}}$ was calculated (-0.29). We suppose that the latter value is inaccurate, because the adsorption layer virtually does not contain SDS at this high DADA fraction, $X_{1 \mathrm{~S}}=0.99$. In the further calculations we use $\beta_{\mathrm{S}}=(-1.31)$, which is determined at $\alpha=0.05$, because the adsorption layer contains almost equal fractions of SDS and DADA at this molar fraction of DADA in the solution (so the precision in the calculation of $\beta_{\mathrm{S}}$ is expected to be the best).
The analysis of the data with respect to the interaction parameter in the micelles, $\beta_{\mathrm{M}}$, was not so straightforward, however. First, we found that $\beta_{\mathrm{M}}=0.20>0$, at low DADA molar fraction $(\alpha=0.05)$, which indicates a net repulsion between the DADA and SDS molecules in the micelles. Second, the calculated value of $\beta_{\mathrm{M}}$ was several times larger at $\alpha=0.09\left(\beta_{\mathrm{M}}=1.12\right)$ as compared to $\alpha=0.05$. Furthermore, no numerical solutions of Eqs. (4) and (6) were obtained at the higher DADA concentrations $(\alpha=0.2$ and 0.7). All these results indicate that there is a net repulsion between the molecules of DADA and SDS in the micelles. This repulsion could probably lead to formation of two types of micelles (one type enriched in SDS molecules, and the other one enriched in DADA molecules) at the higher molar fractions of DADA, which makes questionable the application of the theoretical approach to concentrations above the CMC.

To make further calculations by the method of Ingram [8] for the compositions of the mixed adsorption layers (above the CMC) and of the micelles, we assumed $\beta_{\mathrm{M}}=0.20$ for all mixed micelles. This value of $\beta_{\mathrm{M}}$ corresponds to weak repulsion between the SDS and DADA molecules in the
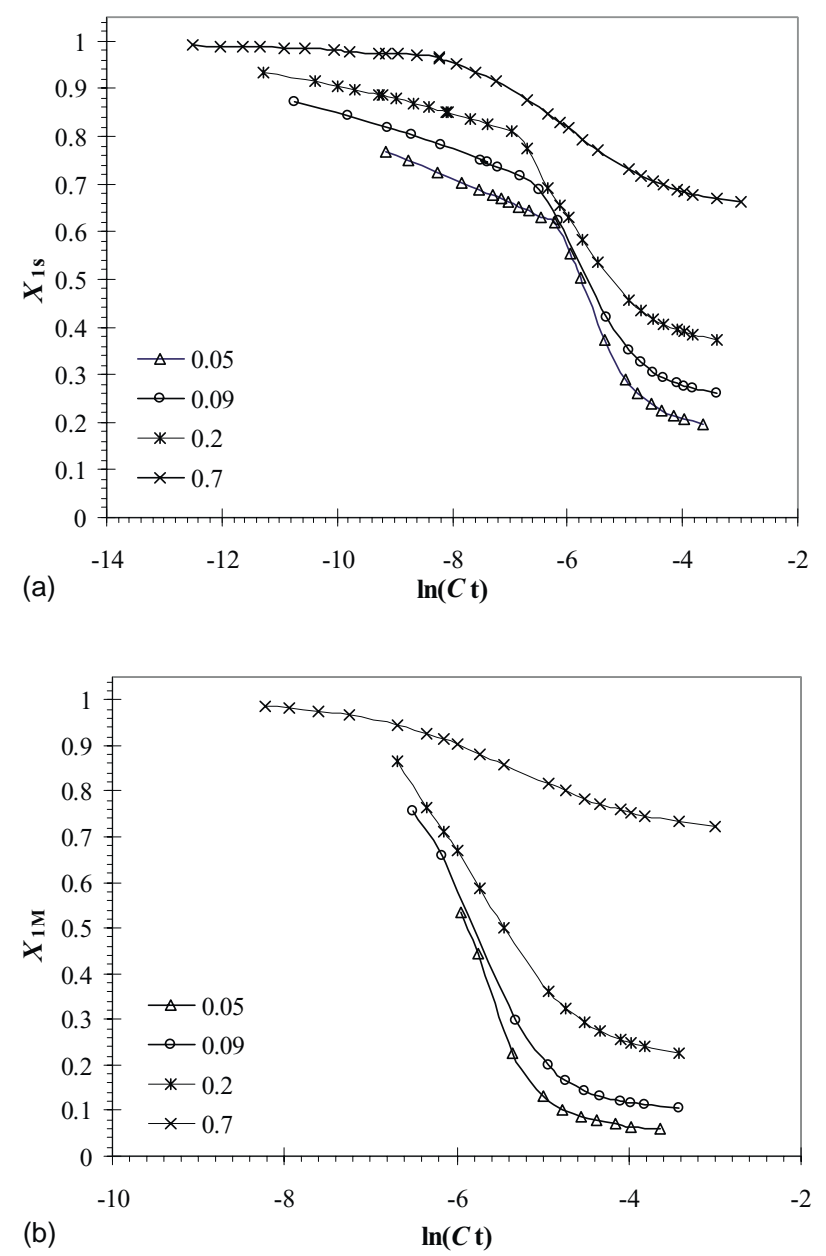

Fig. 5. Composition of the adsorption layers (a) and of the micelles (b) calculated by Ingrams's model, Eqs. (5)-(7). 


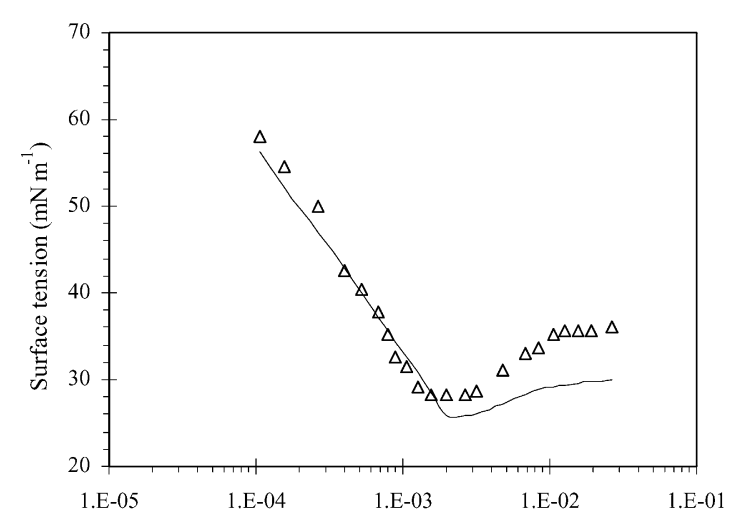

(a)
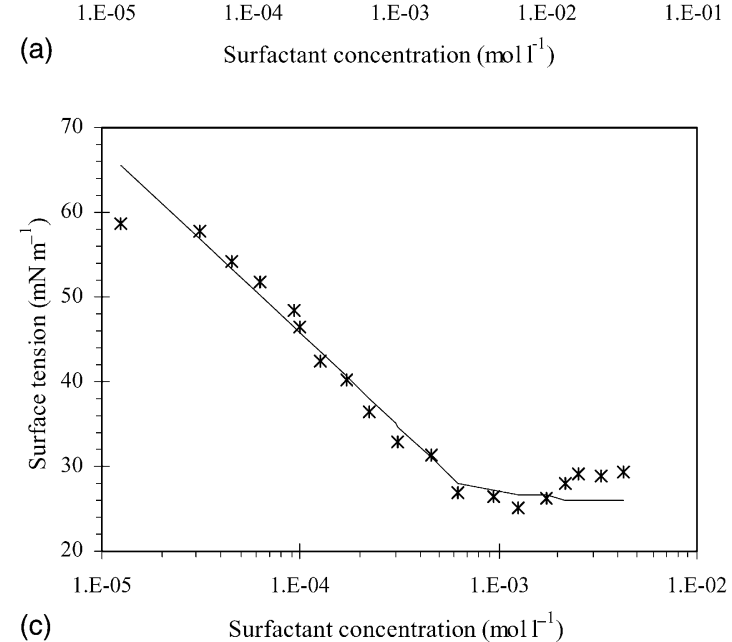

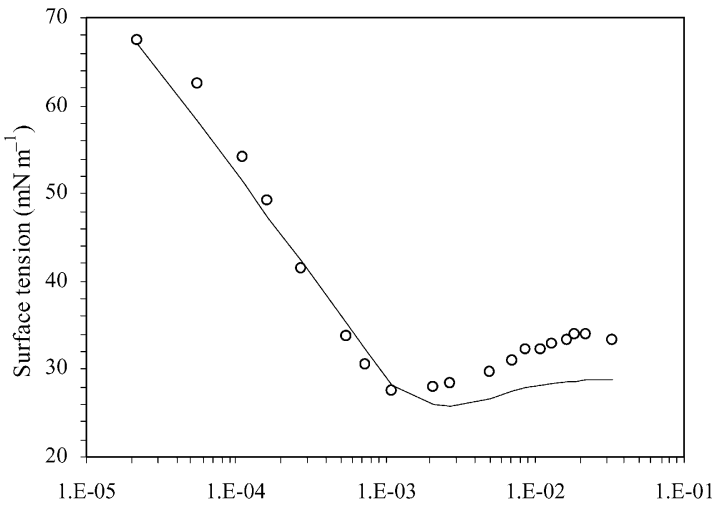

(b)

Surfactant concentration $\left(\mathrm{mol} \mathrm{l}^{-1}\right)$

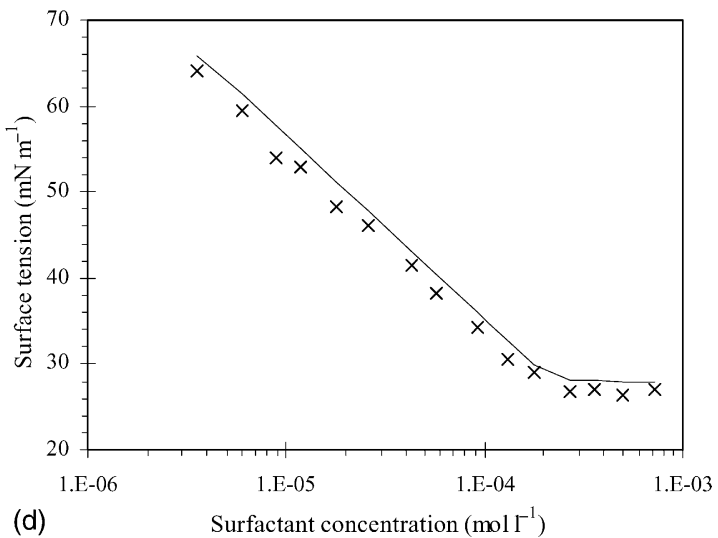

Fig. 6. Plots of experimental (symbols) and theoretically calculated (solid curves) surface tension isotherms of mixed SDS/DADA solutions, containing different molar fractions of DADA: (a) $\alpha=0.05$; (b) $\alpha=0.09$; (c) $\alpha=0.2$; (d) $\alpha=0.7$.

micelles, so that no segregation into two type of micelles is expected (and the theoretical approach is justified). $\beta_{\mathrm{M}}$ was substituted into Eq. (7) and the micelle composition, $X_{1 \mathrm{M}}$, at the studied molar fractions of DADA in the surfactant mixtures $(0.05,0.09,0.2$, and 0.7) were calculated. These values of $X_{1 \mathrm{M}}$ were then substituted into Eq. (6) to yield $C_{1}$ and $C_{2}$ which, in their own turn, were substituted into Eq. (5) to determine $\sigma$ and $X_{1 \mathrm{~S}}$ above the CMC.

All results for the compositions of the mixed adsorption layers and of the mixed micelles, calculated by the method of Ingram [8], are plotted in Fig. 5. It is seen that the DADA molecules prevail on the surface and in the micelles $(>50 \%)$ at concentrations below and around the CMC even when DADA presents a small fraction in the surfactant mixture $(5 \mathrm{~mol} \%)$. At concentrations, which are about 1 order of magnitude above the $\mathrm{CMC}$, the composition of the micelles approaches the composition of the total surfactant mixture. The composition of the surfactant adsorption layer also becomes much closer to the composition of the total surfactant mixture at concentrations well above the CMC. Therefore, there is a significant redistribution of surfactant molecules between the adsorption layers and the micelles with the increase of the total surfactant concentration just above the CMC.

Obviously, the value $\beta_{\mathrm{M}}=0.20$, which was used in the above calculations, is not well justified at molar fractions of
DADA, $\alpha>0.05$. Nevertheless, the comparison between the surface tension isotherms, calculated with $\beta_{\mathrm{M}}=0.20$, and the experimental isotherms showed reasonably good agreement for all studied solutions below CMC (see Fig. 6). Good agreement between the calculated and measured isotherms above CMC was found also for the solutions containing not too low DADA fraction $(\alpha=0.2$ and 0.7$)$. At the lowest DADA fractions in the mixture $(\alpha=0.05$ and 0.09$)$ the calculated and measured isotherms agreed only at surfactant concentrations below the minimum in the isotherms, whereas, the theoretical isotherms were found to lay below the experimental points at higher surfactant concentrations. The most possible reason for this discrepancy between the calculated and the measured isotherms around the minimum in the isotherms is the presence of $\mathrm{DOH}$ in the solutions, which is not taken into account in the NIBM model.

\subsection{Composition of the mixed adsorption layers and micelles calculated by NMMM}

To get some idea about the effect of DOH on the micelle composition in the studied solutions, the adsorption isotherms were interpreted also by the NMMM for a three-component mixture. The parameters $\beta_{i j}$ for the pairs DADA/SDS, DADA/DOH and SDS/DOH were calculated from Eq. (4) by using the isotherms presented in Fig. 3. 
Table 2

Composition of mixed micelles evaluated by NMMM

\begin{tabular}{lllll}
\hline$\alpha_{\text {DADA }}$ & Surfactants & $\alpha_{i}$ & $X_{i \mathrm{M}}$ & $f_{i}$ \\
\hline 0.05 & DADA & 0.0500 & 0.227 & 1.15 \\
& SDS & 0.9485 & 0.766 & 1.00 \\
& DOH & 0.0015 & 0.007 & 0.34 \\
0.09 & DADA & 0.0910 & 0.438 & 1.07 \\
& SDS & 0.9077 & 0.555 & 1.03 \\
& DOH & 0.0014 & 0.007 & 0.46 \\
0.2 & DADA & 0.2000 & 0.857 & 1.01 \\
& SDS & 0.7988 & 0.137 & 1.15 \\
& DOH & 0.0012 & 0.007 & 0.88 \\
0.7 & DADA & 0.7000 & 0.992 & 1.01 \\
& SDS & 0.2985 & 0.005 & 0.85 \\
& DOH & 0.0005 & 0.002 & 1.05 \\
\hline
\end{tabular}

$\alpha_{i}$ is the molar fraction of DADA in the studied solution; $X_{i \mathrm{M}}$, molar fraction of DADA in the mixed micelles at CMC; $f_{i}$, respective activity coefficients.

Part of the isotherm of the mixed solution DADA +0.0015 molar parts DOH was measured and the isotherms of pure SDS and DOH were taken from literature $[10,13]$. The concentration $5 \times 10^{-5} \mathrm{M}$ was accepted as $\mathrm{CMC}$ of $\mathrm{DOH}$, because the formation of surfactant aggregates (lenses) was observed on the solution surface above this concentration. For the micelle interaction parameter between DADA and SDS molecules we used the value, $\beta_{12, \mathrm{M}}=0.20$, which was calculated for molar part of DADA, $\alpha=0.05$ (see Table 1).

Based on the isotherms of the individual surfactants SDS [13] and DOH [10], and the isotherm of SDS + DOH, measured by us, we determined that $\beta_{23}$ (the molecular interaction parameter between SDS and DOH) is equal to $(-1.41)$. From the isotherms of DADA, DOH [10] and DADA+DOH solutions we obtained $\beta_{13}=+0.1$ (i.e. a weak net repulsion between the DADA and DOH molecules was registered). The obtained values of $\beta_{12}, \beta_{23}$ and $\beta_{13}$ were used to solve the set of Eqs. (10) and (11) and the molar parts of the three surfactants in the mixed micelles were determined (see Table 2). From the data presented in Table 2 one sees that the molar part of $\mathrm{DOH}$ in the mixed micelles formed in all studied solutions is rather low $(<0.0015)$. Therefore, the presence of DOH in the solutions is not expected to affect strongly the micelle composition.

On the other hand, the micelle composition calculated by NMMM agrees very well with the results from the NIBM model only for the solutions containing 0.2 and 0.7 molar parts of DADA (compare Tables 1 and 2). The calculations of the micelle composition by the NMMM predict that the micelles should contain predominantly SDS molecules around $\mathrm{CMC}$ in the solutions containing low fractions of DADA ( $\alpha=0.05$ and 0.09 ), whereas the NIBM method predicts that these micelles should contain predominantly DADA molecules. The reasons for this discrepancy are not very clear at the present moment. One possible reason could be the formation of two types of micelles (SDS enriched and DADA enriched) as a result of the net molecular repul- sion between the DADA and SDS molecules and the related problems with the determination of the interaction parameter $\beta_{\mathrm{M}}$ (see Section 4.3). Another possible reason is the presence of DOH in the SDS sample, which causes a minimum in the SDS isotherm, and hence, makes dubious the determination of the CMCs of SDS and its mixtures with DADA at low DADA molar fraction, $\alpha$. Besides, the data from Table 2 suggest that the values of the activity coefficients $f_{1}$ and $f_{2}$ (for DADA and SDS, respectively) are close to unity for all investigated solutions, while the values of $f_{3}$ (for DOH) deviate essentially from unity.

\section{Summary of the main results and conclusions}

The equilibrium surface tension isotherms of the individual SDS and DADA, and of their mixed solutions with $0.05,0.09,0.2$ and 0.7 molar parts of DADA were measured (see Fig. 1) and interpreted by NIBM and NMMM methods. Minimum in the isotherm of the used SDS indicated the presence of dodecanol, whose content was estimated to be $\approx 0.15 \%$ molar parts.

From the performed data interpretation by NIBM method one can conclude:

- Below CMC, the solution surface is occupied predominantly by DADA molecules in all mixed solutions with DADA molar fraction $\alpha \geq 0.05$ (see Fig. 5a). Therefore, very small quantities of DADA change significantly the surface properties of SDS solutions, due to the higher surface activity of DADA.

- Above CMC, the molar part of DADA in the adsorption layer rapidly decreases with the total surfactant concentration, because the DADA molecules are incorporated into the micelles. In this concentration range, the SDS molecules prevail in the adsorption layer at not too high DADA content $(\alpha \leq 0.2)$.

- The obtained value of the surface interaction parameter, $\beta_{\mathrm{S}} \approx-1.3$, implies weak attractive interactions between the SDS and DADA molecules in the adsorption layer. The absence of a strong synergism between DADA and SDS is evidenced also by the fact that the isotherms of the studied mixtures lay between the isotherms of the individual surfactants.

- The DADA molecules prevail in the micelles at CMC and their content increases with increasing the molar part of DADA in the solution (see Fig. 5b). The value of $\beta_{\mathrm{M}}$, obtained for the mixed micelles, is positive which means that there is a net repulsion between the SDS and DADA molecules in the mixed micelles.

- The surface tension isotherms of mixed DADA/SDS solutions were theoretically calculated by Ingram's approach [8]. A good agreement was found between the calculated and measured isotherms below CMC. The observed discrepancy between the calculated and measured isotherms above CMC, at low DADA fraction $(\alpha=0.05$ and 0.09$)$, 
is probably due to the presence of $\mathrm{DOH}$ in the studied samples.

- The micelle interaction parameters, $\beta_{i j}$, were determined by the NMM model for the various pairs of surfactants in the studied solutions (Section 4.4). The results for the micelles composition at moderate and high molar parts of DADA, calculated by NMM model, agree well with those obtained by NIBM. A discrepancy is found for the solution containing low fractions of DADA $(\alpha \leq 0.09)$, which is probably due to the presence of DOH in the used SDS sample.

\section{References}

[1] M.J. Rosen (Ed.), Surfactants and Interfacial Phenomena, second ed., Wiley-International Publication, New York, 1989, Chapter 11, p. 393; Q. Zhou, M.J. Rosen, Langmuir 19 (2003) 4555.
[2] E.G. Lomax (Ed.), Amphotheric Surfactants, Marcel Dekker, New York, 1996, Chapter 1, p. 1.

[3] X. Domingo, in: E.G. Lomax (Ed.), Amphotheric Surfactants, Marcel Dekker, New York, 1996, Chapter 3, p. 75

[4] M.F. Cox, N.F. Borys, T.P. Matson, J. Am. Oil Chem. Soc. 62 (1985) 1139.

[5] E.S. Basheva, S. Stoyanov, N.D. Denkov, K. Kasuga, N. Satoh, K. Tsujii, Langmuir 17 (2001) 969.

[6] M.J. Rosen, X.Y. Hua, J. Colloid Interf. Sci. 86 (1982) 164.

[7] M.J. Rosen, D.S. Murphy, J. Colloid Interf. Sci. 110 (1986) 224.

[8] B.T. Ingram, Colloid Polym. Sci. 258 (1980) 191.

[9] P.M. Holand, D.N. Rubingh, J. Phys. Chem. 87 (1983) 1984.

[10] S.-Y. Lin, Y.-Y. Lin, E.-M. Chen, C.-T. Hsu, C.-C. Kwan, Langmuir 15 (1999) 4370.

[11] D.N. Rubingh, in: K.L. Mittal (Ed.), Solution Chemistry of Surfactants, Plenum Press, New York, 1979, p. 337.

[12] S.P. Harrold, J. Colloid Interf. Sci. 15 (1960) 280.

[13] K. Tajima, M. Muramatsu, T. Sasaki, Bull. Chem. Soc. Japan 43 (1970) 1991.

[14] J.P. Fang, P. Joos, Colloids and Surfaces 65 (1992) 121.

[15] P.A. Kralchevsky, K.D. Danov, V.L. Kolev, G. Broze, A. Mehreteab, Langmuir 19 (2003) 5004-5018. 